\title{
Infrared Thermography (IRT) Applications in Aerospace Research and Development
}

\section{Muralidhar}

Directorate of Non Destructive Evaluation, Defence Research \& Development Laboratory

Kanchanbagh, Hyderabad - 500 058, India

e-mail:dr_c_muralidhar@rediffmail.com

Infrared Thermography (IRT) has gained recently wider acceptance in Aerospace industry due to its speed of inspection, sensitivity, repeatability and larger area coverage. Aerospace Research \& Development area is to deal with wide variety ranging from mission critical components to single shot application devices. Each one has to be dealt separately depending on its functional requirements and its configuration details. Hence, in order to meet their stringent quality requirements, the challenge is often to find a suitable Non Destructive Evaluation (NDE) method for defects analysis, to generate acceptance standards and to assess the performance of various systems/sub-systems.

Generally Non Destructive Evaluation (NDE) methods are employed to find out the structural integrity of the test object without affecting its strength and usefulness. Infrared Thermography (IRT) is one such NDE method based on the heat flow in a material is altered by the presence of anomalies, which causes the localized temperature differences resulting in thermal contrast on the material surface. However, IRT can be used as a standalone method or in combination with other NDE methods for effective investigation.

Unlike other NDE methods, IRT is used in addition to NDE, also finds applications extensively in the areas of preventive maintenance, heat exchangers, transmission lines, missile tracking, night vision, Electrical and Mechanical inspection, Refractory insulation diagnostics, Energy conservation, Leak detection, etc. As the training and experience of an IRT interpreter plays a vital role, IRT has drawn some skepticism due to its inappropriate use, poor interpretation of results and lack of experience in its data analysis and failure to integrate it into overall investigation. In spite of these short comings, IRT is widely used for many applications and indeed is preferred even first.

The paper discusses of employing of IRT in the areas of NDE for defects analysis in metallic and composite components, performance evaluation of thermal barrier coatings, evaluation of heating panels efficiency, performance evaluation of infrared suppression systems, monitoring of static firing tests, Infrared signature analysis, fault diagnosis in Printed Circuit Boards (PCB), and monitoring of tool wear \& tear. The results revealed that IRT is a fast, non-contact, nondestructive, non-invasive and covers larger inspection areas. Further, the plants need not to be shut down during IRT inspection, which saves time and money significantly as compared to other methods. IRT provides thermal profile data for entire test area as compared to point of contact in case of Thermocouples. The salient features of IRT are highlighted.

Keywords: Aerospace, Infrared Thermography, Non Destructive Evaluation, Non-contact, Thermal signature 